\title{
Biomedical Importance of Cocoa (Theobroma cacao): Significance and Potential for the Maintenance of Human Health Sara Ishaq ${ }^{1}$, Laila Jafri ${ }^{2}$
}

${ }^{1}$ Department of Biochemistry, Bahauddin Zakariya University, Multan, Pakistan, sarahishaq1622@gmail.com, ${ }^{2}$ Department of Biochemistry, Faculty of Sciences, Bahauddin Zakariya University, Multan, Pakistan

This is an open access article distributed under the Creative Commons Attribution License, which permits unrestricted use, distribution, and reproduction in any medium, provided the original work is properly cited.

\section{ARTICLE DETAILS}

Article history:

Received 22 January 2017

Accepted 03 February 2017

Available online 05 February 2017

Keywords:

Theobroma cacao, Cocoa, Biomedical importance, Cocoa and human health.

\begin{abstract}
Plants and herbs have been the source of medication for man from centuries ago. Many compounds have been extracted from them for treatment of chronic diseases such as cocaine from cocoa. The Cocoa tree belongs to the family Theobroma, present in South and Central America. It has been found to be associated with cardiovascular health, lowering of blood pressure, platelet activities. Its anti-malarial, anti-oxidant, anti- cough, anti-influenza, anti-diabetic, anti-hypertensive activities have also been reported. Cocoa contains elevated amounts of various flavonoids, for example, catechin, epicatechin enantiomers, and procyanidin B2 and methylxanthines. As it has been used since very late in human medication and has a great ethno- pharmacological importance, hence, it can be used as a medicine in future for the treatment of many chronic diseases
\end{abstract}

\section{Introduction}

From early ages, man has been utilizing common herbs and plants as society medicines, yet it is just since the mid-nineteenth century that genuine endeavors were made to seclude and sanitize the dynamic standards for the treatment of different infirmities. From that point forward, a huge assortment of organically dynamic mixes have been gotten and their structures decided, e.g. morphine from opium, cocaine from coca leaves, and quinine from the bark of the cinchona tree [1]. Cocoa beans were accepted to have fearsome mysterious powers by the old and were routinely utilized as a part of customs, religious services, and healings by clerics. By the 1700 s, cocoa was utilized for restorative purposes to treat various scatters, for example, angina and heart torment [2]. More than 100 restorative uses for cocoa by the old have been recorded in Europe and New Spain from sixteenth to mid twentieth century [3]. Around $80 \%$ of individuals in the world still prefer conventional pharmaceutical construct to a great extent with respect to types of plants and creatures for their essential medicinal services. About 500 plants with therapeutic utilize are said in old writing and around 800 plants have been utilized as a part of indigenous frameworks of pharmaceutical. Home grown solutions as the real cure in conventional arrangement of pharmaceutical have been utilized as a part of medicinal practices since artifact as a result of their biomedical advantages and in addition put in social convictions in many parts of world and have made an awesome commitment towards keeping up human wellbeing. Around $25 \%$ of pharmaceutical remedies in the United States contain no less than one plant-determined fixing. In the most recent century, approximately 121 pharmaceutical items were planned in light of the conventional information acquired from different sources [4].

Pakistan is endowed with a rich abundance of therapeutic plants. Herbs have dependably been major type of prescription in Pakistan and these are getting to be distinctly prevalent all through the created world. Over $70 \%$ of population in Pakistan relies on customary arrangement of medicine. The purpose behind their ubiquity is because of high cost of allopathic medicine and reactions of these solutions. The aggregate estimation of pharmaceuticals expended in nation both foreign made and fabricated by national and multinational medication organizations is more than 100 billion rupees for every year. Out of this, $95 \%$ crude material is transported in. Different research papers demonstrates that all territories of Pakistan in Himalayan range are rich in herbs, which are of high incentive in pharmaceutical enterprises [5].

The name "Cocoa" gets from the Spanish word cacao, got from the Nahuatl word cacahuatl. [6] The Nahuatl word, thus, at last gets from the remade Proto Mije-Sokean word ${ }^{*}$ kakaw ${ }^{*}$ kakawa [7]. The Cocoa tree belongs to the family Theobroma, a gathering of trees which happens in the wild in the Amazon bowl and other tropical territories of South and Central America. There are more than twenty species in the variety yet the cocoa tree, Theobroma cacao is the only one developed broadly [8]. Cocoa plants

respond well to relatively high temperatures, with a maximum annual average of $30-32^{\circ} \mathrm{C}$ and a minimum average of $18-21^{\circ} \mathrm{C}$. Cocoa needs a sensible amount of supplements, to a profundity of $1.5 \mathrm{~m}$ to permit the improvement of a decent root framework. Beneath that level it is attractive not to have impermeable material, with the goal that overabundance water can deplete away. Cocoa will withstand waterlogging for brief periods; however overabundance water ought not to wait. The cocoa tree is touchy to an absence of water, so the dirt must have both water maintenance properties and great waste [9].

A cocoa unit (natural product) has a harsh, rugged skin around 2 to 3 $\mathrm{cm}(0.79$ to $1.18 \mathrm{in}$ ) thick (this shifts with the beginning and assortment of case) loaded with sweet, adhesive mash (called baba de cacao in South America) with a lemonade-like taste encasing 30 to 50 expansive seeds that are genuinely delicate and a pale lavender to dull earthy purple shading (Fig 2.). Because of warmth development in the aging procedure, cacao beans lose the vast majority of the purplish tone and turn out to be for the most part chestnut in shading, with a followed skin which incorporates the dried stays of the fruity mash. This skin is discharged effectively in the wake of simmering by winnowing. White seeds are found in some uncommon assortments, generally blended with purples, and are considered of higher esteem. The three fundamental assortments of cocoa plant are Forastero, Criollo, and Trinitario (Fig 2). The first is the most generally utilized, containing $80-90 \%$ of the world generation of cocoa. Cocoa beans of the Criollo assortment are rarer and considered a delicacy $[10,11]$.

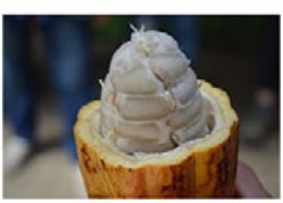

Fig 1.Cocoa beans in freshly cut cocoa pods

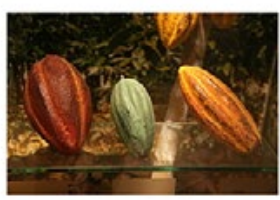

Fig 2. Three main varieties of cocoa Criollo, Trinitario and Forastero

For a long time, chocolate was expended only for pleasure, but in the last 20 years researches have shown that dull chocolate and cocoa could have valuable impact on human health due to high content of polyphenols [12]. Cocoa is rich in polyphenols, like those found in green tea, and as polyphenols have been appeared to have beneficial consequences for CVD [13], cholera, consumption (tuberculosis), scarlet fever, smallpox, typhus, and yellow fever [14], neurodegenerative illnesses, change in lipid digestion system, hindrance of low-thickness lipoprotein (LDL) oxidation, lessening in atherosclerotic injury arrangement restraint of platelet accumulation , diminish in vascular cell grip atom expression, change in endothelial capacity decrease in pulse and hostile to cancer-causing impacts $[15,16]$. (Fig 3.) 


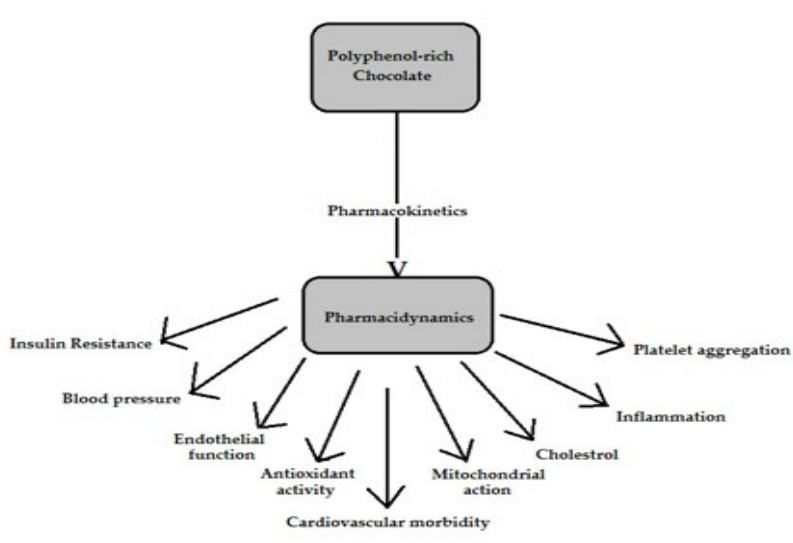

Fig 3. Summary of polyphenols' effects [17]

\section{METHODOLOGY:}

A thorough search was conducted on the electronic databases, Science direct and PubMed/Medline. The main keywords used were 'Cocoa', 'Biomedical Importance of Cocoa', 'Theobroma cacao', 'Health effects of cocoa' and 'Cocoa extracts and their biomedical uses', and the literature was retrieved using search engines mainly google scholar. As the aim was to look for more authentic information, the search was mainly limited to scholarly research articles only. Our specified search as a result of the combination of the previous words showed more than 10,000 results. More than 100 research articles and reviews were extracted, which were analysed according to the inclusion/study selection criteria.

\subsection{Study selection criteria:}

The articles selection was mainly based upon the forthcoming criteria, and the articles published both in English as well as in non-English languages were included in the study. The primary selection of a study was performed based upon its title, which was later on checked for eligibility by all authors after reviewing the abstract followed by the full text. As a result, about 73 articles were selected based on the inclusion/study selection criteria.

\section{NUTRITIONAL VALUE:}

Cocoa powder is an item got from the beans of the Theobroma cacao tree, which is viewed as a decent wellspring of fiber $(26 \%-40 \%)$, proteins $(15 \%-20 \%)$, sugars (around 15\%) and lipids $(10 \%-24 \%$; by and large, $10 \%-12 \%)$. It additionally contains minerals, vitamins and some bioactive mixes $[18,19]$

\section{PHYTOCHEMISTRY:}

Cocoa contains elevated amounts of various flavonoids, for example, catechin epicatechin enantiomers (Fig 4.) [20], procyanidin B2, methylxanthines (Fig 5.) [21], tannins, saponin, cardiac glycosides, terpenoids and alkaloids (Fig 6.) $[22]$.

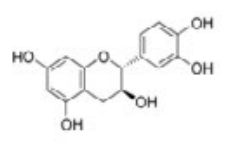

(+)-Catechin (2R, 3S)

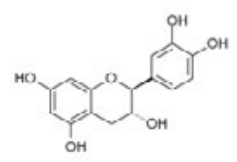

(-)-Catechin (2S, 3R)

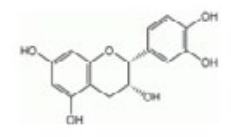

(-)-Epicatechin (2R, 3R)

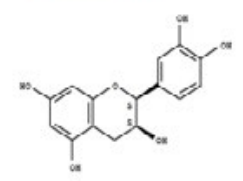

(+)-Epicatechin (2S, 3S)

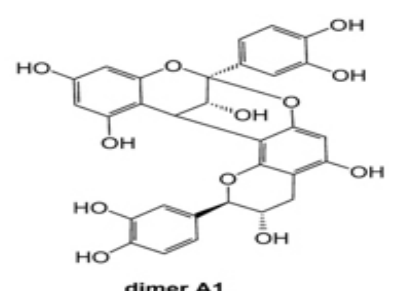

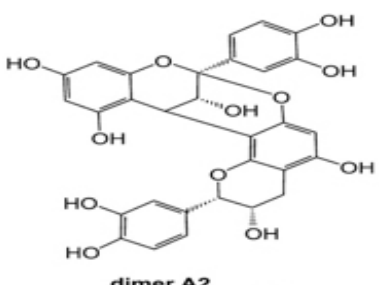<smiles>[R]C1C=C([C@H]2O[C@H]3C[C@@H](O)C[C@H](O)[C@H]3C[C@H]2O)CCC1[R]</smiles>

epicatechin $\left(R_{1}+R_{2}=O H\right)$ 3'-O-methyl-epicatechin $\left(\mathrm{R}_{1}=\mathrm{OCH}_{3}, \mathbf{R}_{2}=\mathrm{OH}\right)$ 4'-O-methyl-epicatechin ( $\left.R_{1}=O H, R_{2}=\mathrm{OCH}_{3}\right)$

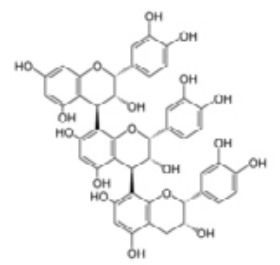<smiles>[R6]O[R6](=O)O[Na]</smiles>

Trimer Cl, epicatechin- $(4 \beta \rightarrow 8)] 2$-epicatechin<smiles>Cn1c(=O)c2c(ncn2C)n(C)c1=O</smiles><smiles>Cn1c(=O)c2[nH]cnc2n(C)c1=O</smiles>

Caffeine

Theophylline<smiles>Cn1cnc2c1c(=O)[nH]c(=O)n2C</smiles>

Theobromine

Fig 5. Structures of procyanidin dimers and trimer, and methylxanthines in cocoa<smiles>O=C(O)c1cc(O)c(O)c(O)c1</smiles><smiles>COC(=O)C1C[C@@H]2CC[C@H](C1)N2C</smiles>

Tannins
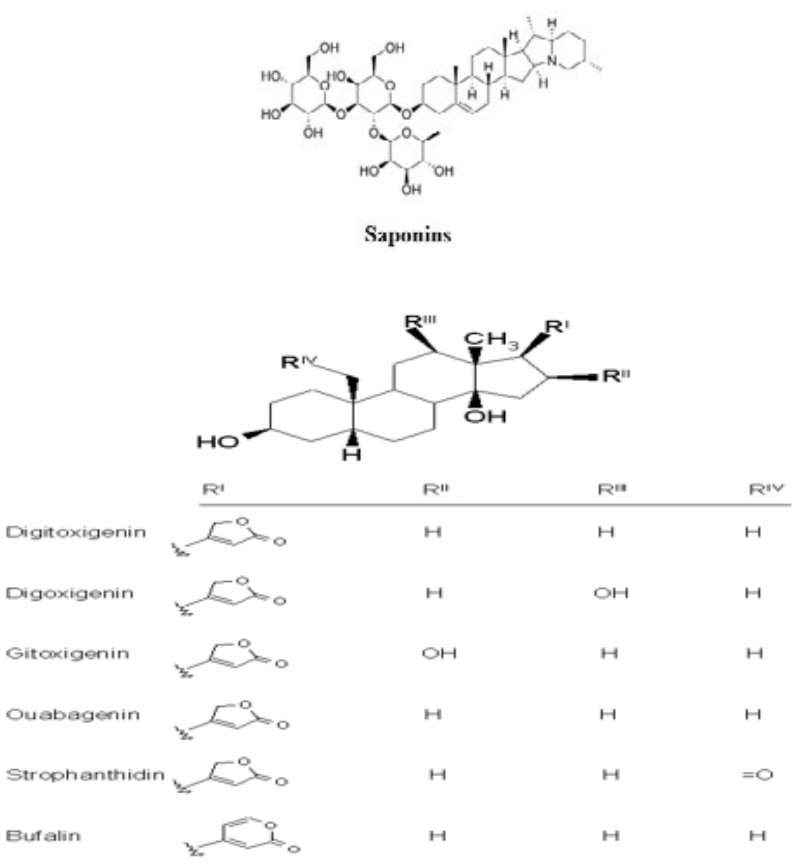

Cardiac glycosides 


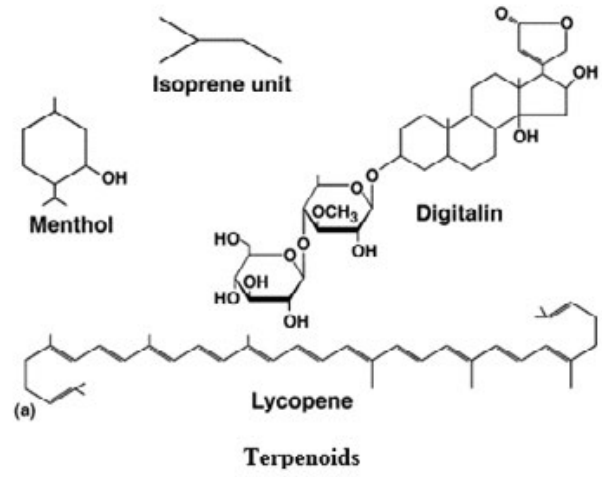

Fig 6. Structures of tannins, saponins, cardiac glycosides, terpenoids and alkaloids.

\section{BIOLOGICAL ACTIVITIES OF THEOBROMA CACAO:}

\subsection{Antioxidant Activity:}

An in vitro and in vivo study was conducted to check the antioxidant activity of cocoa plant. Survey showed that the flavanols from cocoa extracts have a high antioxidant activity [23]. Similarly in another study theaflavin, epigallocatechin gallate, resveratrol, and procyanidin present in cocoa extracts were found to have high antioxidant activity [24]. Likewise the antioxidant activity and phenolic content of four hybrids of cocoa beans was determined by using FRAP assay and it was found out that these hybrids have more phenolic content than traditional cocoa beans and thus more antioxidant activity [25]. In another study the aim was to asses the oxidative status in terms of lipid peroxidation and antioxidant enzymes in different tissues of orally administered cocoa-treated rats Oxidative status was evaluated by Catalase, GSH, SOD, MDA and 4-HNE, in Liver, Heart and Kidney tissues. It was concluded that the beneficial antioxidant effects and cocoa powder enhance the antioxidant enzymes level in liver and heart tissues of rats in group II, while no remarkable changes was observed in kidney tissue [26]. It was found from the in vivo studies and an overview of the current literature that cocoa has high antioxidant activity and useful for skin health [27].

\subsection{Effects on Blood Pressure:}

Five randomized controlled trails of cocoa administration involving a total of 173 subjects with a median duration of 2 weeks were studied. These randomized dietary studies indicated that consumption of foods rich in cocoa may reduce blood pressure [28]. Similarly it was studied whether habitual cocoa intake was cross-sectionally related to blood pressure and prospectively related with cardiovascular mortality. Small,shortterm,intervention studies indicate that cocoa-containing foods improve endothelial function and reduced blood pressure [29]. Likewise another study was conducted on 15 healthy persons according to the World Medical Association's Declaration of Helsinki and it was found out that Dark, but not white, chocolate decreases blood pressure and improves insulin sensitivity in healthy persons [30]. In the same way, data was collected from a group trial involving 44 adults using dark chocolate which indicated that inclusion of small amounts of polyphenol-rich dark chocolate as part of a usual diet efficiently reduced $\mathrm{BP}$ and improved formation of vasodilative nitric oxide [31]. Berry and Davison conducted a randomized, double-blind, cross-over trial in 2010 with cocoa bevarages in of either low-flavanol or high-flavanol content and concluded that cocoa flavanols may decrease blood pressure and also reduce risk factors of cardiac failure [32]. Another study resulted in the concept that (-)-epicatechin can modulate BP in hypertension by increasing NO levels in the vasculature in which the antihypertensive effect of dietary (-)-epicatechin was investigated on spontaneously hypertensive rats (SHRs) [33]. A randomized study showed that daily intake of lycopene-containing (L-tug) lycosome formulation of dark chocolate by healthy prehypertensive individuals with borderline-to-moderate hypercholesterolemia reduces the systemic blood pressure, as well as the total cholesterol and triglycerides in serum after the 1 month observational period [34].

\subsection{Effects on Brain Perfusions:}

A study was designed to investigate the effects of flavanol-rich cocoa (FRC) consumption on cerebral blood flow in older healthy volunteers. Data suggested a promising role for regular cocoa flavanol's consumption in the treatment of cerebrovascular ischemic syndromes, including dementias and stroke as it showed that dietary intake of FRC is associated with a significant increase in cerebral blood flow velocity in the MCA as measured by TCD [35]. It was demonstrated that short-term ingestion of cocoa, particularly rich in the subclass of flavonoids known as flavanols, induced a consistent and striking peripheral vasodilation in healthy people, improving endothelial function in a nitric oxide-dependent manner. Flavanol-poor cocoa induced much smaller responses in healthy older subjects [36]. It was examined that whether 1-year administration of a cocoa polyphenolic extract (Acticoa powder), orally delivered at the dose of $24 \mathrm{mg} / \mathrm{kg}$ per d between 15 and 27 months of age, affects the onset of age-related cognitive deficits, urinary free dopamine levels and lifespan in old Wistar-Unilever rats. The data suggested that Acticoa powder may be beneficial in retarding age-related brain impairments, including cognitive deficits in normal ageing and perhaps neurodegenerative diseases [37]. 4.5 Anti-Cancer Activity:

In vitro investigations suggested that cocoa displayed an effect in decreasing the mutagenicity of carcinogens. Thus, cocoa itself, and by implication, the procyanidins, is perfectly safe and they emphasized beneficial effects through their antioxidant actions [38]. Another study on polyphenols from cocoa, coffee and tea also resulted in anti-cancer activity of these beverages [39]. The studies on fresh of non-edible cocoa plant parts such as cocoa leaf (CL), cocoa bark (CB), cocoa husk (CH), cocoa shell unfermented (CSUF), cocoa shell fermented (CSF), cocoa root (CR), cocoa cherelle (CC) \& cocoa pith (CP) by extracting in methanol solvent, screening against various type of cancer cell lines (breast with estrogen receptor positive (MCF-7), breast with estrogen receptor negative (MDA-MB-231), liver (HepG2), colon carcinoma (HT-29), lung (A549) and cervical (HeLa) cell lines), purified using bioassay guided fractionation and identified active compounds using gas chromatography-mass spectrometry (GCMS), revealed that the cocoa leaf extract presented the highest antiproliferative activity against breast cancer cell line [40]. Similarly diagnosis on death certificates to compare cause-specific death rates from year 2000 to 2004 in mainland and the San Blas islands where only Kuna live showed that the chances of deaths due to cancer in Kuna are much lower than those of other world. It was due to the reason that the Kuna living in the San Blas drink a flavanol-rich cocoa as their main beverage, contributing more than $900 \mathrm{mg} /$ day and thus probably have the most flavonoid-rich diet of any population [41].

\subsection{Effects on Cardiovascular Health:}

In vitro studies in healthy male adults were conducted in which, the ingestion of flavanol-rich cocoa was associated with acute elevations in levels of circulating NO species, an enhanced flow-mediated dilation (FMD) response of conduit arteries, and an augmented microcirculation [42]. In a cross-sectional Dutch Zutphen analysis, cocoa intake was inversely related to blood pressure, and in a prospective analysis, intake was associated with a reduction of cardiovascular and all-cause mortality [43]. A case-control study done in Italy showed that the risk for myocardial infarction was inversely associated to chocolate consumption, reaching a 77\% decrease in risk when comparing the population that ate more than three portions of chocolate per day with the population that consumed less than one. Several dietary intervention studies in humans and animals demonstrated that cocoa and other flavanol-rich foods/ beverages19-21 may exert protective vascular effects [44]. A systematic review and meta-analysis of randomized, controlled trials was performed by Shrime and Bauer in 2011 to evaluate the effect of flavanoid-rich cocoa on cardiovascular risk factors and to assess a dose-response relationship. Data suggested that the consumption of foods rich in polyphenolic compounds, particularly cocoa, may have cardioprotective effects [45]. Another prospective cohort study was conducted on 31823 women without a history of myocardial infarction. It was found that in this population, moderate habitual chocolate intake was associated with a lower rate of heart failure hospitalization or death [46].

\subsection{Effects on Body's Cholestrol Level:}

A randomized, crossover feeding trial was conducted with 42 high risk volunteers. All received $40 \mathrm{~g}$ of cocoa powder in $500 \mathrm{~mL}$ of skimmed milk/day. The study indicated that cocoa decreases the low-density lipopolysaccharides (LDL) cholestrol level but increases the highdensity lipopolysaccharides (HDL) cholestrol level in the body [47]. A comparative study, examined 160 normocholesterolemic and mildly hypercholesterolemic subjects for plasma LDL cholesterol and oxidized LDL concentrations following the intake of different levels of cocoa powder $(13,19.5$, and $26 \mathrm{~g} / \mathrm{d})$. The survey suggested that polyphenolic substances derived from cocoa powder may contribute to a reduction in LDL cholesterol, an elevation in HDL cholesterol, and the suppression of LDL [48]. Similarly another trial on 25 healthy subjects of cocoa products 
supplementation in humans resulted in effects on LDL oxidizability, but not in urinary F2 isoprostanes or markers of inflammation [49]. The effects of cacao liquor polyphenols (CLP) on the susceptibility of LDL to oxidation in hypercholesterolemic rabbits were examined. In hypercholesterolemic rabbits, orally administered CLP was absorbed and distributed to the blood, and the resistance of LDL to oxidation was thereby increased [50]. A review study showed that dark chocolate/cocoa products significantly reduce serum low-density lipoprotein (LDL) and total cholesterol (TC) levels [51].

\subsection{Anti-Fatigue Syndrome Activity:}

A crossover study was conducted comparing high cocoa liquor/polyphenol rich chocolate (HCL/PR) in comparison to simulated iso-calorific chocolate (cocoa liquor free/low polyphenols(CLF/LP)) on fatigue and residual function in subjects with chronic fatigue syndrome. The study suggested that HCL/PR chocolate may improve symptoms in subjects with chronic fatigue syndrome [52].

\subsection{Anti-Cough Activity:}

Two separate laboratory trials were conducted from 2008 to 2011 and from 2003 to 2009. It was concluded that a combination of honey and coffee can be used as an alternative medicine in the treatment of persistent postinfectious cough (PPC) $[53,54]$.

\subsection{Anti-Diabetic Activity:}

Results from clinical trials have suggested that anthocyanidines from barries and flavan-3-ols from green tea and cocoa may lower the risk of type 2 Diabetes Mellitus and cardiovascular diseases [55]. Similarly in another randomized clinical control trial, 100 subjects with type 2 diabetes mellius were included. Data collected, provided a beneficial effect on lipid peroxidation prevention and inflammatory markers in type 2 Diabetic patients [56]. Laboratory experiments on ICR mice indicated that cacao liquor procyanidin (CLPr) is a beneficial food material for improvement of glucose tolerance by promoting glucose transporter 4 (GLUT4) translocation to the plasma membrane of skeletal muscle [57].

\subsection{Effects on Hummoral Immunity:}

In a laboratory experiment, effect of macrophages on NO synthesis was checked after cocoa extract intake. Data suggested that cocoa extract contains a suppressor of NO production in murine macrophages [58]. Similarly another experiment was conducted to evaluate the effect of cocoa and thyme powder separately or in combination on serum biochemical parameters and humoral immune responses in broiler chicks. It was concluded that the application of thyme and cocoa powder, particularly their combination in broiler diets has the potential to improve serum biochemical values but failed to have favourable impact on humoral immune responses of the birds [59].

\subsection{Anti-Hypertensive Activity:}

A natural flavonoid-enriched cocoa powder, commercially named CocoanOX was characterized and tested for a possible antihypertensive effect. The survey showed that this product was very rich in total procyanidins and the effect of its single oral administration in spontaneously hypertensive rats (SHR) could be used as a functional ingredient with antihypertensive effect [60]. A diverse evidence base data suggested that cocoa lowers BP while improving insulin sensitivity, vascular function, and cardiovascular outcomes [61].

\subsection{Anti-Inflammatory Activity:}

The effect of cocoa polyphenolic extract (CPE) on RAW 264.7 macrophage cells sensitized by lipopolysaccharide as in vitro inflammatory model was investigated. Data obtained, suggested that CPE may be used for the treatment of inflammation and it's related-diseases [62]. Similarly there have been studies suggesting that the regular or occasional consumption of cocoa-rich compounds exerts beneficial effects on blood pressure, insulin resistance, vascular damage, and oxidative stress [63].

\subsection{Anti-Influenza Activity:}

A cocoa extract (CE) was prepared by treating defatted cocoa powder with boiling water. The extract demonstrated dose-dependent inhibition of infection in Madin-Darby canine kidney (MDCK) cells infected with human influenza virus A (H1N1, H3N2), human influenza virus B and avian influenza viruses (H5N1, H5N9). CE inhibited viral adsorption to MDCK cells. Animal experiments showed that CE significantly improved survival in mice after intra-nasal administration of a lethal dose of influenza virus [64].

\subsection{Anti-Malarial Activity:}

In vitro activity of natural cocoa powder on the growth of Plasmodium falciparum was determined. Prepared crude methanol extract was partitioned successively with petroleum ether, ethyl acetate, chloroform, and butanol. Direct inhibitory activity of the natural cocoa powder was assessed by culturing extract and fractions with P. falciparum in vitro. The results suggest that natural cocoa powder has measurable direct in vitro inhibitory effect on P. falciparum and support the anecdotal reports of its ability to prevent malaria as a result of regular intake as a beverage [65]. In another work, cocoa bean extract (CBE) was used to synthesize silver nanoparticles (AgNPs) under ambient conditions. studies showed the conspicuous presence of silver in colloidal solution with distinctive crystalline facecentered cubic phase of AgNPs. CBE-AgNPs showed appreciable activities against multi-drug resistant bacteria, producing zones of inhibition of 10-14 mm, and also enhanced activities of ampicillin, cefuroxime, cefixime and erythromycin by $42.9-100 \%$ in synergistic studies. It produced pronounced antimicrobial activities against several bacteria and fungi, when incorporated into emulsion paint. Furthermore CBE-AgNPs showed good larvicidal activities against Anopheles gambiae mosquito larvae with LC50 of $44.37 \mathrm{lg} / \mathrm{ml}$, while it also prevented the coagulation of human blood [66]. A surevey study on effects of cocoa's ingredients on Malaria parasites and illness suggested anti-malarial activities of cocoa flavanoids and their derivatives [67].

\subsection{Effects on Platelet Activities:}

In an experiment on 16 male subjects, it was conclude that trained subjects show decreased activation of stimulated platelets when compared to the sedentary subjects and short-term cocoa polyphenol supplementation did not decrease platelet activity in response to exercise independent of prior training status [68]. Thirty healthy volunteers were randomized in another study to receive white, milk and dark chocolate and assessments on platelet function were undertaken on venous blood samples before and after chocolate consumption. White and milk chocolate had no significant effect on platelets. However dark chocolate inhibited collagen-induced platelet aggregation in platelet rich plasma [69]. Similarly a study peripheral blood was obtained from 30 healthy subjects before and 2 and $6 \mathrm{~h}$ after ingestion of a cocoa beverage $(n=10)$, a caffeine-containing control beverage $(n=10)$, or water $(n=10)$. Platelet activation was measured in terms of expression of activation-dependent platelet antigens and platelet microparticle formation by using fluorescent-labeled monoclonal antibodies and flow cytometry. Primary platelet-related hemostasis was measured with a platelet function analyzer. It was concluded that cocoa consumption suppressed ADP- or epinephrine-stimulated platelet activation and platelet microparticle formation [70]. In a crossover design, 16 healthy adults consumed aspirin (81 mg), cocoa (as a beverage), or aspirin plus cocoa. Platelet activation was measured by surface expression of P-selectin and PAC-1 binding to the activated conformation of the GPIIb/IIIa receptor (GPIIb/IIIa-act). Platelet function was measured on an analyzer (the PFA-100k) that measures shear stress-induced platelet plug formation in response to collagen-epinephrine or collagen-ADP. It was concluded that flavanol-rich cocoa inhibited epinephrine-stimulated platelet activation and function. These effects were qualitatively similar to aspirin, but less profound [71]. The effect of flavonoidrich dark chocolate was assessed, compared with cocoa-free control chocolate on coronary vascular and platelet function in 22 heart transplant recipients in a double-blind, randomized study. It was concluded that dark chocolate induces coronary vasodilation, improves coronary vascular function, and decreases platelet adhesion 2 hours after consumption. These immediate beneficial effects were paralleled by a significant reduction of serum oxidative stress and were positively correlated with changes in serum epicatechin concentration [72]. Similarly, twenty healthy subjects (HS) and 20 smokers were randomly allocated to receive $40 \mathrm{~g}$ of dark (cocoa $>85 \%$ ) or milk chocolate (cocoa $<35 \%$ ) in a cross-over, single-blind study. There was an interval of 7 days between the two phases of the study. At baseline and $2 \mathrm{~h}$ after chocolate ingestion, platelet recruitment (PR), platelet ROS, platelet isoprostane 8-ISO-prostaglandin F2a (8-iso-PGF2a), Thromboxane (TxA2) and platelet activation of NOX2, the catalytic sub-unit of NADPH oxidase, and serum epicatechin were measured. It was concluded that dark chocolate inhibits platelet function by lowering oxidative stress only in smokers; this effect seems to be dependent on its polyphenolic content [73].

\section{CONCLUSION:}

Theobroma cacao belongs to the Theobroma family, commonly known as cocoa, and is an evergreen tree native to Amazon fuana America. It has been 
used as a folk remedy for persistent post-infectious cough, influenza, high blood pressure, inflammation, flu etc. Its anticancer, antioxidant, antimalarial, antidiabetic, and antihypertensive activities are also reported and it contains phytochemicals such as flavonols, procyanidins, methylxanthines, fibers, and various phenolic compounds. Alongwith, its toxicological effects are either not reported or very negligible. But to best of my knowledge, as such, no medicine has been developed from cocoa yet. Therefore, this review strongly recommends that attention should be given to cocoa and compounds extracted from cocoa to develop useful medicines from them to cure above mentioned and many other chronic diseases. In future, the utilization of cocoa and derived medications in reciprocal form can lessen toxicities and expand restorative results. Not only the treatments, but rather the entire framework itself should work in a reciprocal, moral and comprehensive path for general society to get the most savvy and beneficial mending choices.

\section{REFERENCES}

1. Patrick, G.L., An introduction to medicinal chemistry. 2013: Oxford university press.

2. Dillinger, T.L., et al., Food of the gods: cure for humanity? A cultural history of the medicinal and ritual use of chocolate. The Journal of nutrition, 2000. 130(8): p. 2057S-2072S

3. Keen, C.L., Chocolate: food as medicine/medicine as food. Journal of the American College of Nutrition, 2001. 20(sup5): p. 436S-439S.

4. Verma, S. and S. Singh, Current and future status of herbal medicines. Veterinary world, 2008. 1(11): p. 347-350.

5. Ahmad, S.K.a.D.S., Opportunities and Potentials for Herbal Pharmaceutical Products Development in Pakistan Managing Natural Resources for Sustaining Future Agriculture 2011. 2(19).

6. Bingham, A. and J. Roberts, South and Meso-American mythology A to Z. 2010: Infobase Publishing.

7. Kaufman, T. and J. Justeson, The history of the word for "cacao" and related terms in ancient Meso-America. Chocolate in Mesoamerica: a cultura history of cacao. Gainesville: University Press of Florida, 2006: p. 118-39.

8. Wood, G.A.R. and R. Lass, Cocoa. 2008: John Wiley \& Sons.

9. Almeida, A.-A.F.d. and R.R. Valle, Ecophysiology of the cacao tree. Brazilian Journal of Plant Physiology, 2007. 19(4): p. 425-448.

10. Zipperer, P. and H. Schaeffer, The Manufacture of Chocolate and Other Cacao Preparations. 1915: M. Krayn.

11. Yanamoto, K., H. Sasai, and A. Inoue, Method of producing white cacao nibs and food using white cacao nibs. 1995, Google Patents.

12. Ackar, D., et al., Cocoa polyphenols: can we consider cocoa and chocolate as potential functional food? Journal of chemistry, 2013. 2013.

13.Cooper, K.A., et al., Cocoa and health: a decade of research. British Journal of Nutrition, 2008. 99(01): p. 1-11.

14. Pucciarelli, D.L. and L.E. Grivetti, The medicinal use of chocolate in early North America. Molecular nutrition \& food research, 2008. 52(10): p. 12151227.

15. Vauzour, D., et al., Polyphenols and human health: prevention of disease and mechanisms of action. Nutrients, 2010. 2(11): p. 1106-1131.

16. Lamuela-Raventós, R., et al., Review: health effects of cocoa flavonoids. Revista de Agaroquimica y Tecnologia de Alimentos, 2005. 11(3): p. 159 176.

17. Valussi, M. and C. Minto, Cacao as a Globalised Functional Food: Review on Cardiovascular Effects of Chocolate Consumption. The Open Agriculture Journal, 2016.10(1)

18. Lipp, M., et al., Composition of genuine cocoa butter and cocoa butter equivalents. Journal of Food Composition and analysis, 2001. 14(4): p. 399408.
19. Torres-Moreno, M., et al., Nutritional composition and fatty acids profile in cocoa beans and chocolates with different geographical origin and processing conditions. Food chemistry, 2015. 166: p. 125-132.

20. Kofink, M., M. Papagiannopoulos, and R. Galensa, (-)-Catechin in cocoa and chocolate: occurence and analysis of an atypical flavan-3-ol enantiomer Molecules, 2007. 12(7): p. 1274-1288.

21. Jalil, A.M.M. and A. Ismail, Polyphenols in cocoa and cocoa products: is there a link between antioxidant properties and health? Molecules, 2008 13(9): p. 2190-2219.

22. Subhashini, R., et al., A comparative phytochemical analysis of cocoa and green tea. Indian Journal of Science and Technology, 2010. 3(2): p. 188-192. 23. Keen, C.L., et al., Cocoa antioxidants and cardiovascular health. The American journal of clinical nutrition, 2005. 81(1): p. 298S-303S.

24. Lee, K.W., et al., Cocoa has more phenolic phytochemicals and a higher antioxidant capacity than teas and red wine. Journal of agricultural and food chemistry, 2003.51(25): p. 7292-7295.

25. Jonfia-Essien, W., et al., Phenolic content and antioxidant capacity of hybrid variety cocoa beans. Food chemistry, 2008. 108(3): p. 1155-1159.

26. Noori, S., K. Nasir, and T. Mahboob, Effects of cocoa powder on oxidant/ antioxidant status in liver, heart and kidney tissues of rats. J Anim Plant Sci, 2009. 19: p. 174-8.

27. Scapagnini, G., et al., Cocoa bioactive compounds: significance and potential for the maintenance of skin health. Nutrients, 2014. 6(8): p. 32023213.

28. Taubert, D., R. Roesen, and E. Schömig, Effect of cocoa and tea intake on blood pressure: a meta-analysis. Archives of internal medicine, 2007. 167(7): p. 626-634.

29. Buijsse, B., et al., Cocoa intake, blood pressure, and cardiovascular mortality: the Zutphen Elderly Study. Archives of internal medicine, 2006. 166(4): p. 411-417.

30. Grassi, D., et al., Short-term administration of dark chocolate is followed by a significant increase in insulin sensitivity and a decrease in blood pressure in healthy persons. The American journal of clinical nutrition, 2005. 81(3): p. 611-614.

31. Taubert, D., et al., Effects of low habitual cocoa intake on blood pressure and bioactive nitric oxide: a randomized controlled trial. Jama, 2007. 298(1): p. 49-60.

32. Berry, N.M., et al., Impact of cocoa flavanol consumption on blood pressure responsiveness to exercise. British Journal of Nutrition, 2010. 103(10): p. 1480-1484.

33. Galleano, M., et al., (-)-Epicatechin reduces blood pressure and improves vasorelaxation in spontaneously hypertensive rats by NO-mediated mechanism. IUBMB life, 2013. 65(8): p. 710-715.

34. Petyaev, I.M., et al., Reduction in blood pressure and serum lipids by lycosome formulation of dark chocolate and lycopene in prehypertension. Food science \& nutrition, 2014. 2(6): p. 744-750.

35. Sorond, F.A., et al., Cerebral blood flow response to flavanol-rich cocoa in healthy elderly humans. Neuropsychiatric disease and treatment, 2008. 4(2): p. 433.

36. Fisher, N.D., F.A. Sorond, and N.K. Hollenberg, Cocoa flavanols and brain perfusion. Journal of cardiovascular pharmacology, 2006. 47: p. S210-S214. 37. Bisson, J.-F., et al., Effects of long-term administration of a cocoa polyphenolic extract (Acticoa powder) on cognitive performances in aged rats. British Journal of Nutrition, 2008. 100(01): p. 94-101.

38. Weisburger, J.H., Chemopreventive effects of cocoa polyphenols on chronic diseases. Experimental Biology and Medicine, 2001. 226(10): p. 891-897.

39. Ferrazzano, G.F., et al., Anti-cariogenic effects of polyphenols from plant stimulant beverages (cocoa, coffee, tea). Fitoterapia, 2009. 80(5): p. 255262 